\title{
COMPONENTE DI FONDO E COMPONENTI CICLICHE DELL'INDICE DELLA PRODUZIONE INDUSTRIALE IN ITALIA
}

\author{
MARIO FALIVA(*), MARIA GRAZIA ZOIA(*) \\ Nota presentata dal s.c. Mario Faliva \\ (Adunanza del 26 Marzo 2015)
}

Sunto. - La nota analizza l'Indice della Produzione Industriale (IPI) in Italia esaminandone le evoluzioni temporali tendenziale e congiunturale. L'individuazione delle componenti di fondo e cicliche della serie storica IPI è condotta con un apparato analitico che fa perno su una approssimazione discreta finita di un filtro passa-basso ideale. L'andamento di fondo che emerge è indicativo di un processo di crisi in atto, di carattere strutturale, operante nel paese dai primi anni del nuovo millenio.

Abstract. - The note is meant to investigate the Italian Index of Industrial Production (IIP) for both the long- and the short-run. To this end, the trend and the cyclic components are detected from the parent IIP time series via a signal-theory argument, which hinges on a finite discrete approximation of an ideal low-pass filter.

The behavior of the long-run component thus obtained provides impressive evidence of the structural nature of the crisis afflicting the Italian economy since the beginning of the new millennium.

(*) Dipartimento di Discipline matematiche, Finanza matematica ed Econometria dell'Università Cattolica del Sacro Cuore, Milano, Italia.

E-mail: mario.faliva@unicatt.it ; maria.zoia@unicatt.it 
1. La serie storica dell'indice della produzione industriale e le sue componenti

La crisi che attraversa in questi anni l'Europa, e l'Italia in particolare, ha secondo gli esperti una natura non semplicemente congiunturale ma strutturale.

L'individuazione e l'analisi con tecniche appropriate delle componenti di fondo e ciclica dell'indice della produzione industriale nel nostro paese (vedi grafico sottostante) può condurre ad una valutazione e ad una diagnosi del fenomeno.

Figura 1 - Serie grezza dell'Indice della produzione industriale in Italia.

Dati trimestrali, periodo $1^{\circ}$ trimestre $1948-3^{\circ}$ trimestre 2014 , base $2010=100$

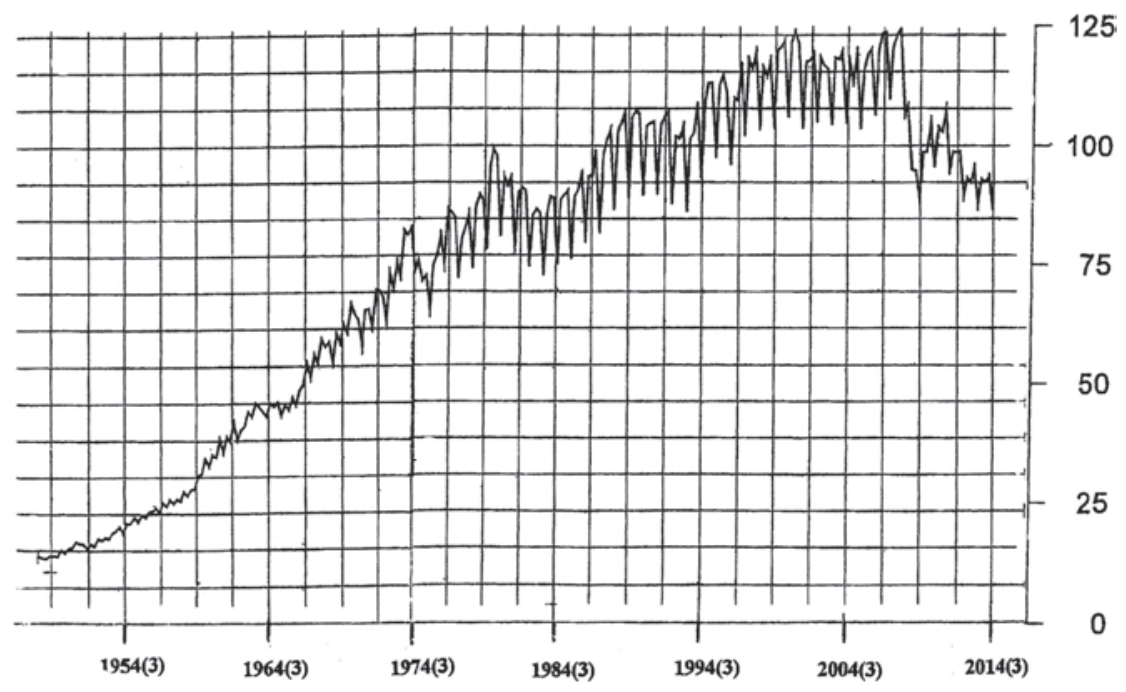

Fonte ISTAT

Come è noto, una serie storica economica è interpretabile come la risultante di componenti (non osservabili) di lungo, medio e breve periodo - e specularmente di bassa, media e alta frequenza, rispettivamente - denominate componente di fondo (trend), componente ciclica e componente stagionale, a cui si sovrappone una componente accidentale (cfr. Faliva, 1978).

Il modello che collega la serie storica osservata alle sue componenti (non osservabili) può essere così formulato

$$
x_{t}=f_{t}+c_{t}+s_{t}+\eta_{t}
$$


dove:

$x_{t}$ sta ad indicare la serie storica osservata $(t=1,2, \ldots, \mathrm{N})$;

$f_{t}$ sta ad indicare la componente di fondo o trend;

$c_{t}$ sta ad indicare la componente ciclica;

$s_{t}$ sta ad indicare la componente stagionale;

$\eta_{t}$ sta ad indicare la componente accidentale.

Nell'accezione comunemente accolta, la nozione di componente di fondo (trend) di una serie storica economica è quella di componente regolare di lungo periodo.

La componente all'oggetto si connota per un andamento monotonico o unimodale nel periodo di osservazione della serie, risultante di componenti permanenti o comunque persistenti su archi temporali adeguatamente lunghi (che nel seguito assumeremo comunque non inferiori a dodici anni).

La nozione di ciclo è quella di fluttuazioni di durata pluriennale - con periodicità variabili, comprese (coniugando teoria economica ed evidenza empirica) fra i tre ed i dodici anni - e con escursioni che si manifestano con ampiezze mutevoli nel tempo (fenomeno denominato evolutività, ed interpretabile alla stregua di un "segnale" modulato, cfr. Papoulis, 1962). Col termine ciclo, o ciclo economico, si indica la cosidetta componente congiunturale.

In economia si opera di norma una distinzione fra cicli medio/lunghi, noti come cicli di Juglar (cfr. Korotayev e Tsirel, 2010), e cicli medio/brevi, noti come cicli di Kitchin (ibid.). Nel seguito converremo di trattare come medio-brevi i cicli dai tre ai sei anni, e come medio-lunghi quelli dai sei ai dodici.

La componente stagionale corrisponde alle oscillazioni su base annuale. L'ampiezza dell'escursione può mutare da un anno all'altro (fenomeno dell'evolutività già riscontrato per il ciclo).

La componente accidentale ha la natura di un rumore di fondo che si sovrappone alle altre componenti introducendo un elemento di disturbo. 


\section{Bande di frequenze e filtri: dalla serie osservata alle sue componenti}

Volendo disporre di una chiave di lettura che permetta di interpretare l'evoluzione di un sistema economico partendo dai dati rilevati, si deve poter enucleare le componenti (non osservabili) di fondo e cicliche dalle serie storiche osservate al fine di disporre delle informazioni salienti sulle dinamiche tendenziali e congiuntuali dei fenomeni allo studio, depurando le serie grezze dalle componenti indesiderate, stagionale e accidentale.

La possibilità di stimare correttamento gli andamenti tendenziale e ciclico di una serie storica economica poggia crucialmente su una reinterpretazione della serie e delle sue componenti in chiave frequenziale (cfr. Faliva e Zoia, 2013; Faliva, 1978).

La gamma di frequenze angolari $\omega$ di pertinenza si colloca fra $-\pi$ e $\pi$. Supporremo nel seguito che la cadenza di rilevazione dei dati sia trimestrale, ovvero che si disponga di $\mathrm{T}=4$ osservazioni per anno.

Con questi assunti, alla componente di fondo risultano essere associate le basse frequenze, ovvero le frequenze nella banda $|\omega|<\frac{2 \pi}{12 T}=\frac{\pi}{24}$, quale immagine speculare delle nozioni di permanenza o comunque di lunga durata nell'accezione datane nel paragrafo precedente (con dodici anni quale valore di soglia).

Alla componente ciclica sono associate le frequenze intermedie, e più specificatamente le frequenze nella banda $\frac{\pi}{24}<|\omega| \leq \frac{2 \pi}{3 T}=\frac{\pi}{6}$, in conformità con la nozione di ciclo precedentemente accolta (cfr. paragrafo 1), che copre periodi fra tre e dodici anni.

Alla componente stagionale sono associate le (bande di) frequenze disposte attorno a $|\omega|=\frac{\pi}{2}$ ed a $|\omega|=\pi$, e come tali "alte" rispetto alle precedenti.

La componente accidentale, in quanto assimilabile ad un rumore di fondo, può ritenersi spalmata pressoché uniformemente sulla gamma di frequenze $-\pi<\omega \leq \pi$.

La collocazione delle componenti nel dominio delle frequenze (con riferimento al semiasse positivo) può essere visualizzata come indicato nella figura che segue. 
Figura 2 - Collocazione delle componenti nel dominio delle frequenze

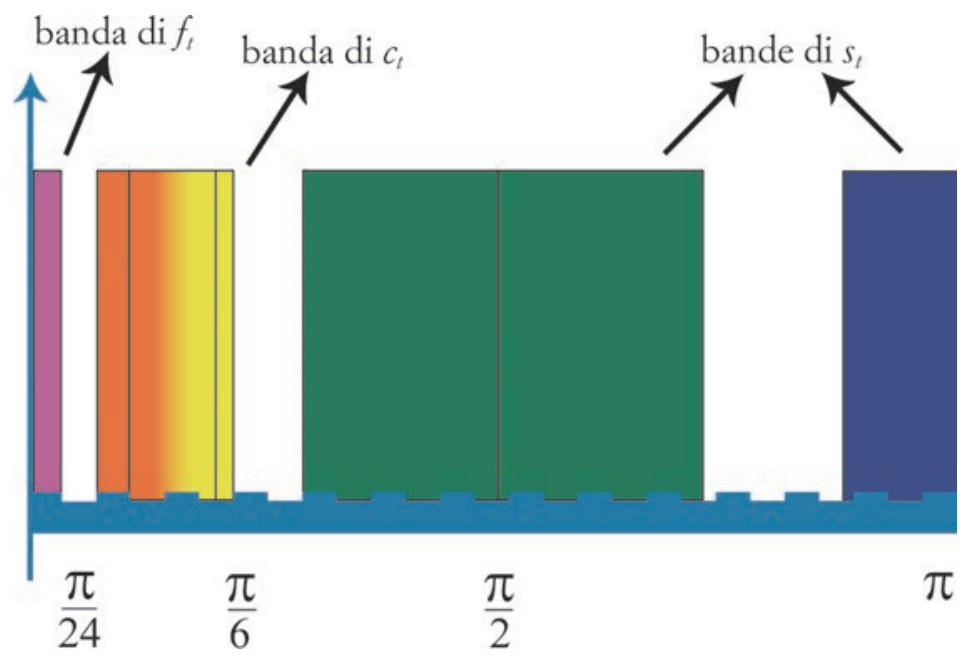

L'ottica frequenziale è la chiave che consente di dare una formulazione rigorosa al problema della enucleazione delle componenti di interesse come problema di filtraggio sulla banda delle frequenze medio-basse al fine di estrarre il "segnale" corrispondente al ciclo-trend.

Formalmente la trasformazione che, agendo sulla serie grezza, permette di estrarre il ciclo-trend può essere rappresentata (ignorando la componente accidentale) come qui di seguito illustrato.

Figura 3 - Dalla serie osservata al ciclo-trend nell'ottica dei filtri

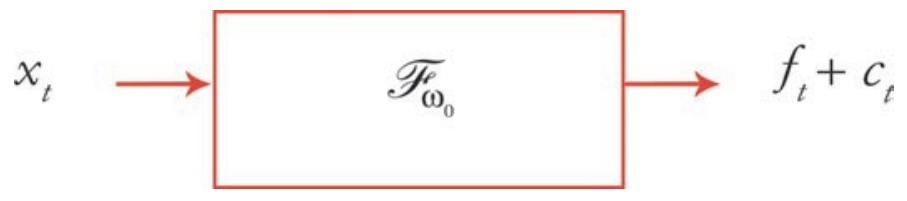

Nella figura sopra $\widetilde{F}_{\omega_{0}}$ sta ad indicare il filtro passa-basso ideale con frequenza di taglio $\omega_{0}$, e più specificatamente $\omega_{0}=\frac{\pi}{6}$, corrispondente alla frequenza di soglia superiore del ciclo-trend. 
In termini formali scriveremo

$$
\mathscr{F}_{\frac{\pi}{6}}\left(x_{t}\right) \simeq f_{t}+c_{t}
$$

dove il simbolo $\simeq$ sta per uguaglianza a meno della componente di disturbo.

Il comportamento del filtro $\frac{\mathscr{F}_{\frac{\pi}{6}}}{6}$ nel dominio delle frequenze è descritto dalla sua funzione di trasferimento $\lambda(\omega)$ che nel caso specifico assume la forma indicata nella figura che segue (il riferimento è al solo semi-asse positivo delle frequenze per semplicità).

Figura 4 - Funzione di trasferimento del filtro ideale

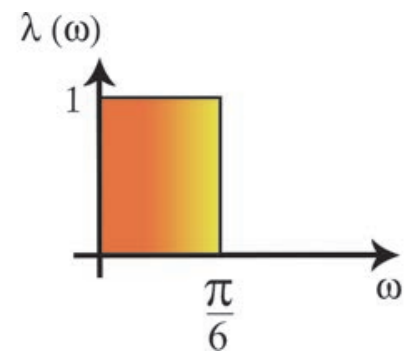

Il filtro $\mathscr{F}_{\omega_{0}}$ ideale (o comunque assimilabile a quello ideale, per tener conto delle esigenze operative) con frequenza di taglio $\omega_{0}=\frac{\pi}{6}$ lascia passare (senza distorsione significativa) le componenti della serie $x_{t}$ inerenti alla banda di frequenze del ciclo-trend, mentre blocca (o comunque attenua drasticamente) le componenti di alta frequenza, e specificatamente la stagionalità.

Per la enucleazione della sola componente di fondo si può procedere in maniera del tutto analoga ricorrendo ad un filtro passa-basso con frequenza di taglio $\omega_{1}=\frac{\pi}{24}$, corrispondente alla frequenza di soglia superiore del trend. 
Indicando con $\mathscr{\mathscr { F }}_{\omega_{0}}$ e con $\mathscr{F}_{\omega_{1}}$ i due filtri passa-basso volti alla enucleazione del ciclo-trend e della componente di fondo, rispettivamente, possiamo formalmente scrivere

$$
\begin{gathered}
f_{t}+c_{t} \simeq \mathscr{F}_{\omega_{0}}\left(x_{t}\right), \omega_{0}=\frac{\pi}{6} \\
f_{t} \simeq \mathscr{F}_{\omega_{1}}\left(x_{t}\right), \omega_{1}=\frac{\pi}{24}
\end{gathered}
$$

Ne consegue altresì che $c_{t}$ può essere determinato come segue

$$
c_{t} \simeq \underset{\frac{\pi}{6}}{\mathscr{F}}\left(x_{t}\right)-\underset{\frac{\pi}{24}}{\mathscr{\mathscr { F } ^ { P }}}\left(x_{t}\right)
$$

Per estensione volendo enucleare dalla serie grezza i cicli medio-lunghi, $c_{t(m l)}$, distintamente dai cicli medio-brevi, $c_{t(m b)}$, ciò si può ottenere ricorrendo ad un ulteriore filtro passa-basso $\mathscr{F}_{\omega_{2}}$ con frequenza di taglio $\omega_{2}=\frac{\pi}{12}$, corrispondente alla frequenza di demarcazione fra le due tipologie di cicli.

Formalmente le componenti desiderate risultano così espresse

$$
\begin{aligned}
& c_{t(m l)} \simeq \mathscr{F}_{\frac{\pi}{12}}\left(x_{t}\right)-\mathscr{F}_{\frac{\pi}{24}}\left(x_{t}\right) \\
& c_{t(m b)} \simeq \underset{\frac{\pi}{6}}{\mathscr{F}}\left(x_{t}\right)-\mathscr{F}_{\frac{\pi}{12}}\left(x_{t}\right)
\end{aligned}
$$

Partendo da queste premesse, che configurano il quadro teorico e metodologico di riferimento, nel prossimo paragrafo potremo procedere alla stima delle componenti tendenziale e congiunturale della serie dell'indice della produzione industriale in Italia. Ciò sarà reso possibile grazie ad un recente contributo degli autori di questa memoria che ha condotto alla identificazione e caratterizzazione di filtri che operano virtualmente come filtri ideali con serie di numerosità finita (cfr. Barbieri, Faliva e Zoia. 2013). 


\section{L'individuazione delle componenti di fondo e congiunturali dll'indice della produzione industriale}

Indicheremo nel seguito con $\mathbf{x}$ il vettore colonna di $\mathrm{N}$ osservazioni sul fenomeno allo studio (nel prosieguo l'indice della produzione industriale) nell'arco temporale $\mathrm{t}=1,2, \ldots, \mathrm{N}$ coperto dalle rilevazioni statistiche, indicheremo con $\mathbf{f}, \mathbf{c}, \mathbf{c}_{\mathrm{m},}, \mathbf{c}_{\mathrm{mb}}$, i vettori delle componenti di $\mathbf{x} \mathrm{e}$ con $\hat{\mathbf{f}}, \hat{\mathbf{c}}, \hat{\mathbf{c}}_{\mathrm{ml}}, \hat{\mathbf{c}}_{\mathrm{mb}}$, i vettori delle relative stime.

Ai sensi di quanto esposto nel paragrafo precedente, il procedimento per enucleare dalla serie di partenza le componenti desiderate comporterebbe il ricorso a filtri passa-basso ideali con frequenze di taglio appropriate.

Alla nozione di filtro passa-basso con frequenza di taglio $\omega_{0}$ può essere associata una matrice di Toeplitz $\sum\left(\omega_{0}\right)$ che, applicata (moltiplicata per) alla serie di riferimento, enuclea dalla stessa la componente desiderata o più precisamente la sua stima. In particolare

$$
\begin{aligned}
& \sum\left(\frac{\pi}{6}\right) \mathbf{x}=\hat{\mathbf{f}}+\hat{\mathbf{c}} \\
& \sum\left(\frac{\pi}{24}\right) \mathbf{x}=\hat{\mathbf{f}}
\end{aligned}
$$

Stante che al filtro ideale corrisponde una matrice $\sum\left(\omega_{0}\right)$ di dimensioni infinite i cui elementi ne rispecchiano la risposta impulsiva (ovvero la trasformata di Fourier della funzione di trasferimento $\lambda(\omega))$ il ricorso al filtro ideale è precluso in tutti i casi concreti in cui le serie si compongono di un numero $\mathrm{N}$ finito di osservazioni.

E grazie ad un recente contributo degli autori che è stata dimostrata la possibilità di costruire filtri con matrice $\sum_{\mathrm{N}}\left(\omega_{0}\right)$ di dimensioni $\mathrm{NxN}$ finite che operano virtualmente come filtri ideali con serie di lunghezza finita N. Rinviando a Barbieri, Faliva e Zoia (2013) per gli aspetti tecnici e le caratteristiche del filtro effettivo, diamo nella figura sottostante la rappresentazione della funzione di trasferimento virtuale del filtro all'oggetto, indicato nel seguito come filtro FZ. 
Figura 5 - Funzione di trasferimento del filtro FZ

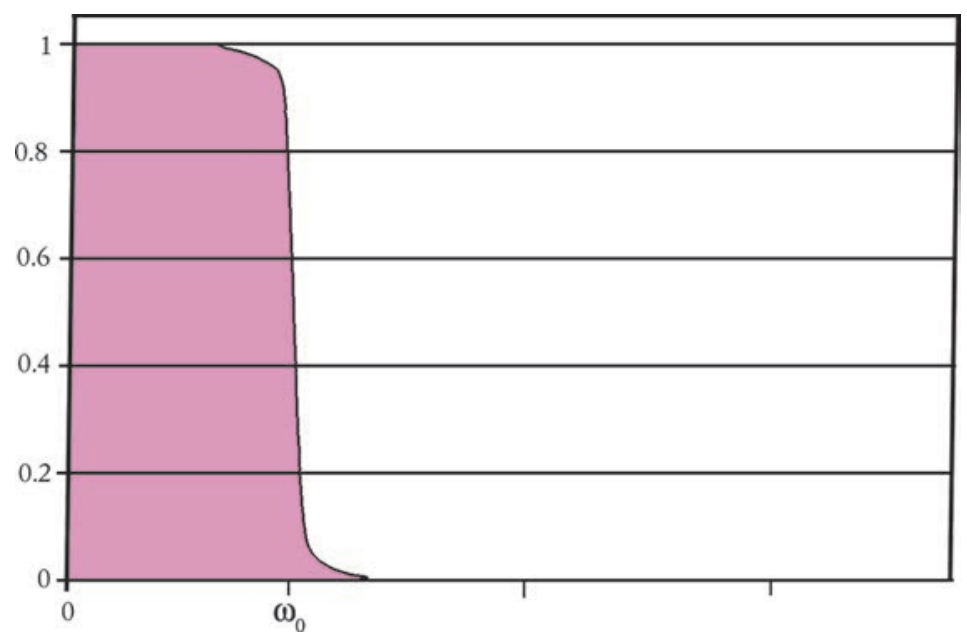

Con riferimento all'Indice della produzione industriale in Italia di cui alla Fig. 1 del $₫ 1$, costruendo le matrici $\sum_{N}\left(\omega_{1}\right)$ dei filtri FZ con frequenze di taglio $\frac{\pi}{6}, \frac{\pi}{24}, \frac{\pi}{12}$, per $\mathrm{N}=267$, che corrisponde al numero di dati rilevati dal $1^{\circ}$ trimestre 1948 al $3^{\circ}$ trimestre 2014 , le componenti stimate $\hat{\mathbf{f}}+\hat{\mathbf{c}}, \hat{\mathbf{f}}, \hat{\mathbf{c}}, \hat{\mathbf{c}}_{\mathrm{ml}}, \hat{\mathbf{c}}_{\mathrm{mb}}$, sono esprimibili come qui di seguito indicato

i) Stima della componente ciclo-trend col filtro FZ con frequenza di taglio $\omega_{0}=\frac{\pi}{6}$ per serie di numerosità $\mathrm{N}=267$ :

$$
\hat{\mathbf{f}}+\hat{\mathbf{c}}=\sum_{N}\left(\frac{\pi}{6}\right) \mathbf{x}, N=267
$$

L'andamento della componente stimata $\hat{\mathbf{f}}+\hat{\mathbf{c}}$ così ottenuta è rappresentato graficamente nella figura sottostante 
Figura 6 - Grafico della componente ciclo-trend stimata dell'Indice della produzione industriale in Italia

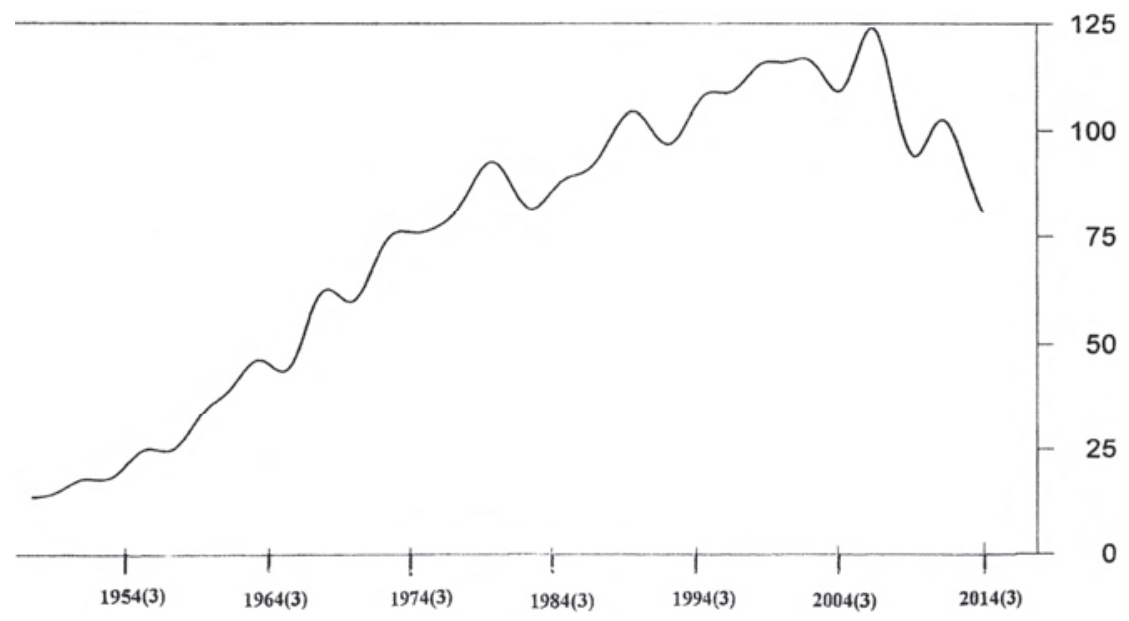

ii) Stima della componente di fondo con filtro FZ con frequenza di taglio $\omega_{1}=\frac{\pi}{24}$, per serie di numerosità $\mathrm{N}=267$ :

$$
\hat{\mathbf{f}}=\sum_{N}\left(\frac{\pi}{24}\right) \mathbf{x}, N=267
$$

L'andamento della componente stimata $\hat{\mathbf{f}}$ così ottenuta è rappresentato graficamente nella figura sottostante 
Figura 7 - Grafico della componente di fondo stimata dell'Indice della produzione industriale

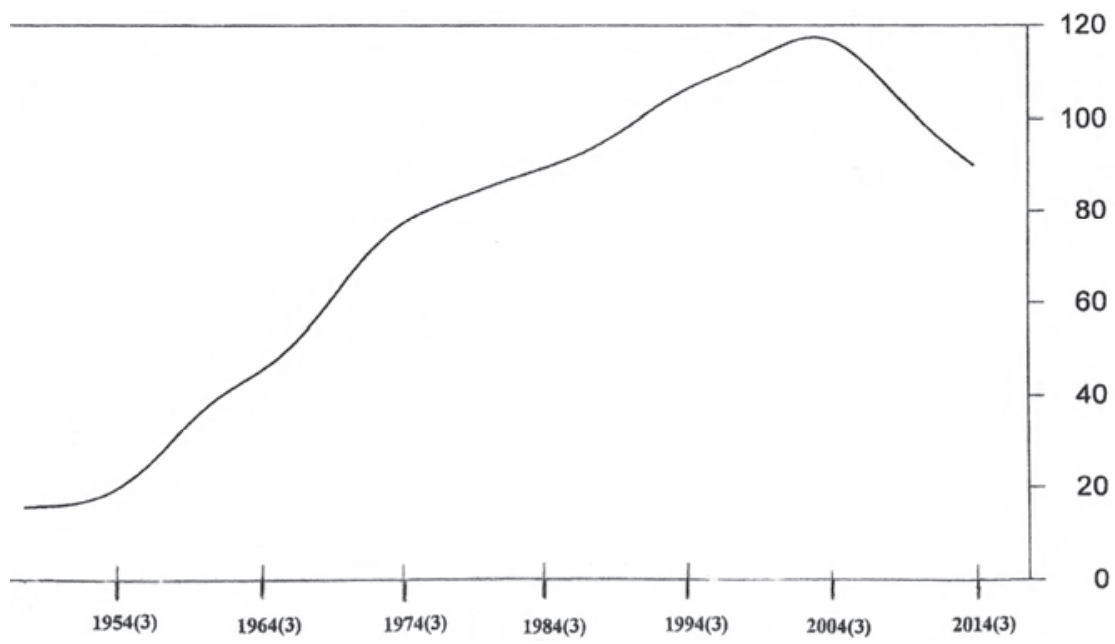

iii) Stima della componente congiunturale col ricorso combinato ai filtri FZ con frequenze di taglio $\omega_{0}=\frac{\pi}{6}$ e $\omega_{1}=\frac{\pi}{24}$ per serie di numerosità $\mathrm{N}=267$ :

$$
\hat{\mathbf{c}}=\sum_{\mathrm{N}}\left(\frac{\pi}{6}\right) \mathbf{x}-\sum_{\mathrm{N}}\left(\frac{\pi}{24}\right) \mathbf{x}, \mathrm{N}=267
$$

L'andamento della componente stimata $\hat{\mathbf{c}}$ così ottenuta è rappresentato graficamente nella figura sottostante. 
Figura 8 - Grafico del ciclo stimato dell'Indice della produzione industriale

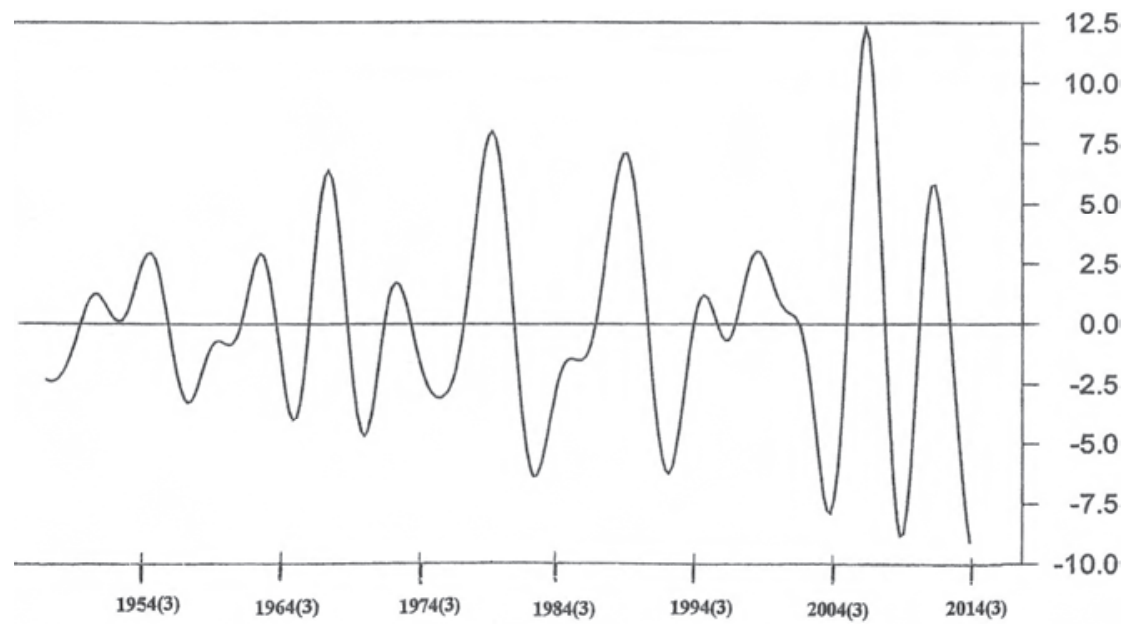

iv) Stima della componente ciclica medio-breve col ricorso combinato ai filtri FZ con frequenze di taglio $\omega_{0}=\frac{\pi}{6}$ e $\omega_{2}=\frac{\pi}{12}$ per serie di numerosità $\mathrm{N}=267$ :

$$
\hat{\mathbf{c}}_{\mathrm{mb}}=\sum_{\mathrm{N}}\left(\frac{\pi}{6}\right) \mathbf{x}-\sum_{\mathrm{N}}\left(\frac{\pi}{12}\right) \mathbf{x}, \mathrm{N}=267
$$

L'andamento della componente stimata $\hat{\mathbf{c}}_{\mathrm{mb}}$ così ottenuta è rappresentato graficamente nella figura sottostante. 
Figura 9 - Grafico del ciclo medio-breve stimato dell'Indice della produzione industriale

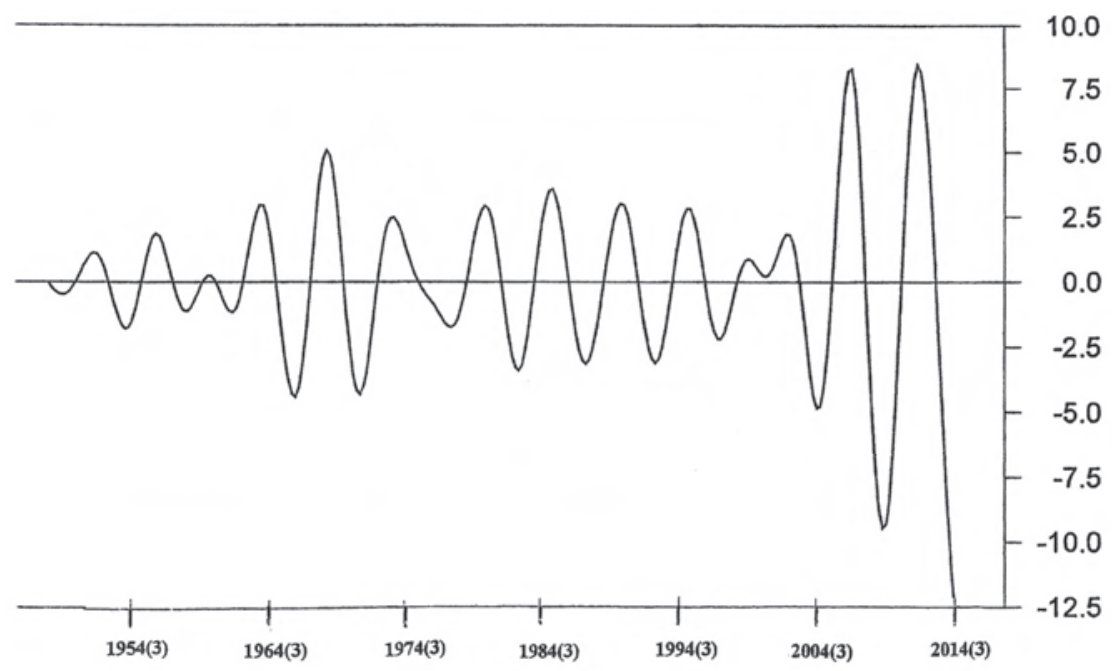

v) Stima della componente ciclica medio-lunga col ricorso combinato ai filtri FZ con frequenze di taglio $\omega_{2}=\frac{\pi}{12}$ e $\omega_{1}=\frac{\pi}{24}$ per serie di numerosità $\mathrm{N}=267$ :

$$
\hat{\mathbf{c}}_{\mathrm{ml}}=\sum_{\mathrm{N}}\left(\frac{\pi}{12}\right) \mathbf{x}-\sum_{\mathrm{N}}\left(\frac{\pi}{24}\right) \mathbf{x}
$$

L'andamento della componente stimata $\hat{\mathbf{c}}_{\mathrm{ml}}$ così ottenuta è rappresentato graficamente nella figura sottostante 
Figura 10 - Grafico del ciclo medio-lungo stimato dell'Indice della produzione industriale

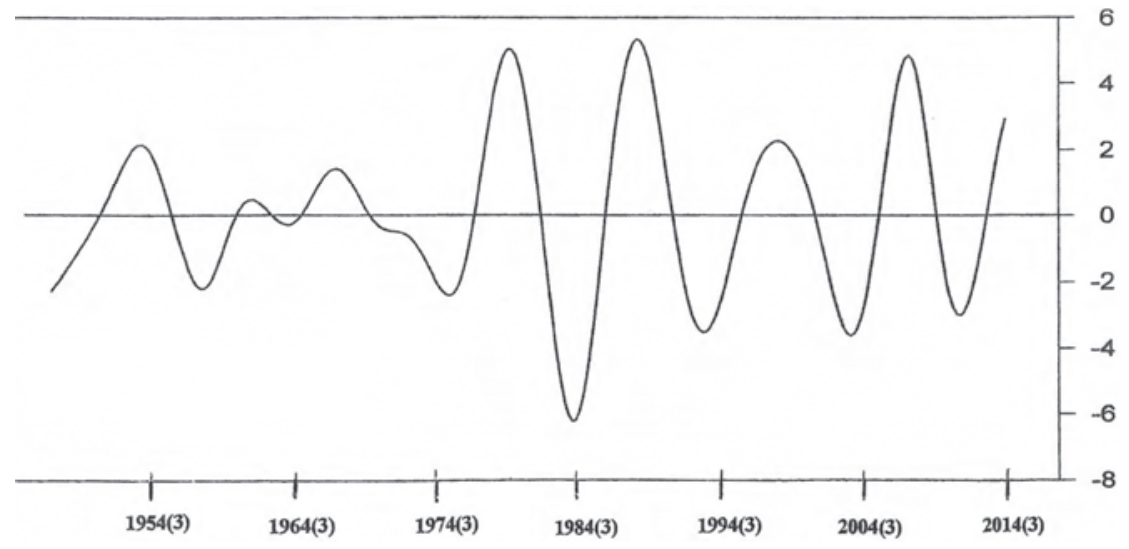

L'esame delle componenti stimate a seguito della procedura di filtraggio di cui alle figure precedenti permette di cogliere - nei tratti salienti - le dinamiche di lungo, medio e breve periodo che presiedono all'andamento dell'Indice della produzione industriale nel nostro Paese nel recente passato.

I grafici più informativi per una visione di sintesi sullo stato di "salute" dell'economia italiana, sotto il profilo della produzione industriale, sono quelli del ciclo-trend (Figura 6) e della componente di fondo (Figura 7). Quest'ultima merita un'ulteriore analisi che possa favorire una migliore percezione del fenomeno ed orientarne la diagnosi.

A tal fine enucleiamo dalla Figura 7 la parte relativa alle ultime tre decadi, che coprono il periodo 1984-2014. Il grafico che ne risulta è riportato nella figura sottostante 
Figura 11 - Componente di fondo stimata dell'Indice della produzione industriale in Italia dagli anni '80 al 2014

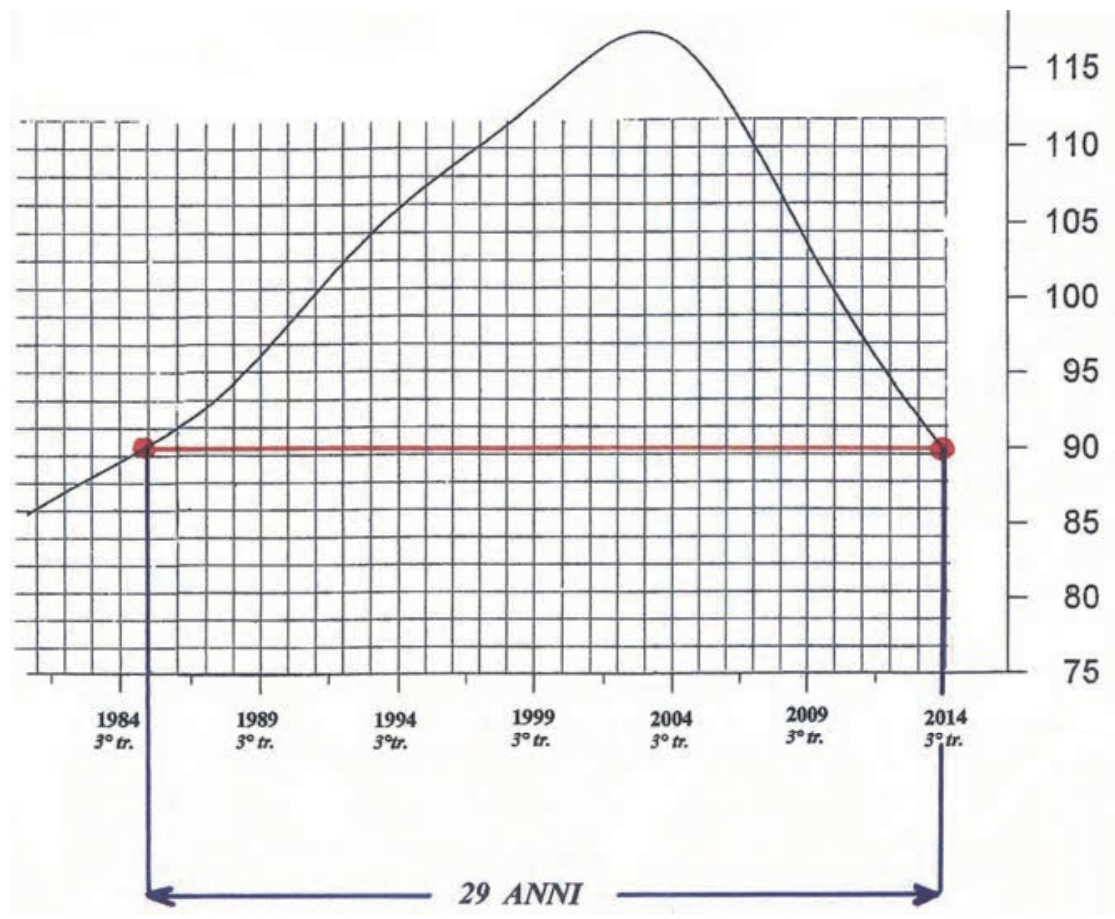

Da ispezione del grafico - ovvero dai dati ottenuti a seguito della procedura di filtraggio ai sensi della formula (3.4) - emerge un fatto di innegabile gravità: la Produzione industriale in Italia oggi (inteso come il trimestre dell'ultimo dato disponibile al momento della presentazione della memoria, $3^{\circ}$ trimestre 2014) è alla stesso livello di 29 anni prima (ovvero del 1985).

La diagnosi, per cruda che possa sembrare, è poco consolante ed è che il Paese è affetto da una crisi strutturale, per la quale non bastano periodiche dichiarazioni confortanti su più o meno favorevoli congiunture favorevoli delle esportazioni o di settori di eccellenza per l'immagine del Paese.

Esula dagli intenti (e dalle competenze) dei relatori pronunciarsi sulle "terapie". Certo è che di fronte ad an andamento come quello registrato nel grafico di Figura 11, non basteranno cure palliative per poter invertire una tendenza, che sembra più rammentare scenari post-bellici 
piuttosto che il naturale decorso delle attività economiche in un contesto normale, pur con i suoi alti e bassi

\section{Riferimenti bibliografici}

Barbieri L., Faliva M., Zoia M.G. (2013), Band-limited component estimation in time-limited economic series, Journal of Applied Statistics 40, 2009-2023.

Faliva M. (1978), Optimal filtering for seasonal adjustment of quarterly data, Rivista internazionale di Scienze Sociali LXXXVI, 55-86.

Faliva M., Zoia M.G. (2013), Econometria: istanze dell'economia e parallelismi con le scienze naturali, in L'Economia quantitativa diventerà una tecnologia del futuro?, a cura di Nicola P.C., Zanella A., Bianchi A.D., Istituto Lombardo di Scienze e Lettere, Milano, 91-102.

Korotayev A.V., Tsirel S.V. (2010), A spectral analysis of world GDP dynamics: Kondratieff waves, Kuznets swings, Juglar and Kitchin cycles in global economic development, and the 2008-2009 economic crisis, Structure and Dynamics 4, 1-55.

Papoulis A. (1962), The Fourier Integral and its Applications, McGraw-Hill, New York. 